\title{
MIXED TYPE OF ADDITIVE AND QUINTIC FUNCTIONAL EQUATIONS
}

\author{
Abasalt Bodaghi, Pasupathi Narasimman, Krishnan Ravi, Behrouz \\ SHOJAEE
}

\begin{abstract}
In this paper, we investigate the general solution and Hyers-UlamRassias stability of a new mixed type of additive and quintic functional equation of the form

$$
\begin{aligned}
f(3 x+y)-5 f(2 x+y)+f(2 x-y)+10 f(x+y)-5 f(x-y) & \\
= & 10 f(y)+4 f(2 x)-8 f(x)
\end{aligned}
$$
\end{abstract}

in the set of real numbers.

\section{Introduction}

In 1940, Ulam [17] raised the following question. Under what conditions does there exist an additive mapping near an approximately addition mapping? The case of approximately additive functions was solved by Hyers [7] under the assumption that for $\epsilon>0$ and $f: E_{1} \rightarrow E_{2}$ with

$$
\|f(x+y)-f(x)-f(y)\| \leq \varepsilon
$$

Received: 17.07.2014. Revised: 31.10.2014.

(2010) Mathematics Subject Classification: 39B72, 39B82.

Key words and phrases: additive functional equation, Hyers-Ulam stability, quintic functional equation. 
for all $x, y \in E_{1}$, then there exist a unique additive mapping $T: E_{1} \rightarrow E_{2}$ such that $\|f(x)-T(x)\| \leq \varepsilon$ for all $x \in E_{1}$, where $E_{1}$ is a normed space and $E_{2}$ is a Banach space.

In 1978, a generalized version of the theorem of Hyers for approximately linear mapping was given by Th.M. Rassias [15]. He proved that for a mapping $f: E_{1} \rightarrow E_{2}$ for which $f(t x)$ is continuous in $t \in \mathbb{R}$ and for each fixed $x \in E_{1}$, there exist constant $\varepsilon>0$ and $p \in[0,1)$ with

$$
\|f(x+y)-f(x)-f(y)\| \leq \varepsilon\left(\|x\|^{p}+\|y\|^{p}\right)
$$

for all $x, y \in E_{1}$, then there exist a unique linear mapping $T: E_{1} \rightarrow E_{2}$ such that

$$
\|f(x)-T(x)\| \leq \frac{2 \varepsilon}{2-2^{p}}\|x\|^{p}
$$

for all $x \in E_{1}$. A number of mathematicians were attracted by the result of Th.M. Rassias (see also [1], [3], [6], [11, 8], 9], [10] and [16]). The stability concept that was introduced and investigated by Rassias is called the HyersUlam-Rassias stability.

In 1982-1989, J.M. Rassias [13, 14 replaced the sum appeared in right hand side of the equation (1.1) by the product of powers of norms.

In [18], Xu et al. obtained the general solution and investigated the Ulam stability problem for the following quintic functional equation

$$
\begin{array}{r}
f(x+3 y)-5 f(x+2 y)+10 f(x+y)-10 f(x)+5 f(x-y)- \\
=120 f(x-2 y) \\
=120
\end{array}
$$

in quasi- $\beta$-normed spaces via fixed point method. This method which is different from the "direct method", initiated by Hyers in [7, had been applied by Cădariu and Radu for the first time. In other words, they employed this fixed point method to the investigation of the Cauchy functional equation [5] and for the quadratic functional equation [4.

In [12], Park et al. introduced the following new form of quintic functional equations

$$
\begin{aligned}
f(3 x+y)-5 f(2 x+y)+f(2 x-y) & +10 f(x+y)-5 f(x-y) \\
& =10 f(y)+f(3 x)-3 f(2 x)-27 f(x) .
\end{aligned}
$$

They applied the fixed point method to establish the Hyers-Ulam stability of the orthogonally quintic functional equation 1.2 in Banach spaces and in non-Archimedean Banach spaces (see also [2]). 
In this paper, we prove the general solution and Hyers-Ulam-Rassias stability of the new mixed additive and quintic functional equation of the form

$$
\begin{array}{rl}
f(3 x+y)-5 f(2 x+y)+f(2 x-y)+10 & f(x+y)-5 f(x-y) \\
& =10 f(y)+4 f(2 x)-8 f(x)
\end{array}
$$

in real numbers. It is easily verified that the function $f(x)=\alpha x^{5}+\beta x$ is a solution of the functional equation 1.3 .

\section{Main Results}

Throughout this paper, we denote the set of real number by $\mathbb{R}$. Before proceeding the proof of main results in this section, we shall need the following lemma.

Lemma 2.1. The only nonzero solution $f: \mathbb{R} \rightarrow \mathbb{R}$ such that $f(x)=g(x)+$ $h(x)$ admitting a finite limit of the quotient $\frac{g(x)}{x}$ and $\frac{h(x)}{x^{5}}$ at zero, of the equation 1.3. is of the form $g(x)=a x$ and $h(x)=b x^{5}$ for all $x \in \mathbb{R}$.

Proof. Putting $y=0$ in (1.3), we have

$$
f(3 x)-8 f(2 x)+13 f(x)=0
$$

for all $x \in \mathbb{R}$. Replacing $(x, y)$ by $(x, x)$ in 1.3 and using 2.1 , we get

$$
f(4 x)-34 f(2 x)+64 f(x)=0
$$

for all $x \in \mathbb{R}$. Setting $g(x)=f(2 x)-32 f(x)$ in 2.2 , we obtain

$$
g(2 x)=2 g(x)
$$

for all $x \in \mathbb{R}$. The relation 2.3 implies that

$$
\frac{g(x)}{x}=\lim _{n \rightarrow \infty} \frac{g\left(\frac{x}{2^{n}}\right)}{\frac{x}{2^{n}}}=a
$$

for some $a \in \mathbb{R}$. Note that $a$ cannot be zero, otherwise, we will have $g=0$. Now, setting $h(x)=f(2 x)-2 f(x)$ in $(2.2)$, we get

$$
h(2 x)=32 h(x)
$$


for all $x \in \mathbb{R}$. It follows from 2.4 that

$$
\frac{h(x)}{x^{5}}=\lim _{n \rightarrow \infty} \frac{h\left(\frac{x^{5}}{2^{n}}\right)}{\frac{x}{2^{n}}}=b
$$

for some $b \in \mathbb{R}$, as claimed. Clearly, $b$ cannot vanishes. This completes the proof.

From now on, we use the abbreviation for the given mapping $f: \mathbb{R} \rightarrow \mathbb{R}$ as follows:

$$
\begin{aligned}
\mathcal{D}_{q} f(x, y)= & f(3 x+y)-5 f(2 x+y)+f(2 x-y)+10 f(x+y) \\
& -5 f(x-y)-10 f(y)-4 f(2 x)+8 f(x)
\end{aligned}
$$

TheOREM 2.2. Let $\phi: \mathbb{R} \times \mathbb{R} \rightarrow[0, \infty)$ be a mapping such that

$$
\sum_{j=0}^{\infty} \frac{1}{2^{j}} \phi\left(2^{j} x, 2^{j} y\right)<\infty
$$

for all $x, y \in \mathbb{R}$ in which $x \in\{x, 0\}$ and $y \in\{x,-x\}$. Suppose that a mapping $f: \mathbb{R} \rightarrow \mathbb{R}$ with $f(0)=0$ satisfies the inequality

$$
\left|\mathcal{D}_{q} f(x, y)\right| \leq \phi(x, y)
$$

for all $x, y \in \mathbb{R}$. Then the limit

$$
A(x)=\lim _{n \rightarrow \infty} \frac{1}{2^{n}}\left\{f\left(2^{n+1} x\right)-32 f\left(2^{n} x\right)\right\}
$$

exists for all $x \in \mathbb{R}$ and the mapping $A: \mathbb{R} \rightarrow \mathbb{R}$ is a unique additive mapping satisfying

$$
|f(2 x)-32 f(x)-A(x)| \leq \frac{1}{2} \sum_{n=0}^{\infty} \frac{\psi\left(2^{n} x\right)}{2^{n}}
$$

for all $x \in \mathbb{R}$ where

$$
\psi(x)=\phi(x, x)+\frac{25}{2} \phi(0, x)+5 \phi(x,-x) .
$$


Proof. Replacing $(x, y)$ by $(0, x)$ in $(2.6)$, we get

$$
|f(x)+f(-x)| \leq \frac{1}{4} \phi(0, x)
$$

for all $x \in \mathbb{R}$. Letting $x=y$ in $(2.6)$, we have

$$
|f(4 x)-5 f(3 x)+6 f(2 x)-f(x)| \leq \phi(x, x)
$$

for all $x \in \mathbb{R}$. Substituting $(x, y)$ by $(x,-x)$ in $(2.6)$, we obtain

$$
|-8 f(2 x)+f(3 x)+3 f(x)-10 f(-x)| \leq \phi(x,-x)
$$

for all $x \in \mathbb{R}$. It follows from $2.10-2.12$ that

$$
|f(4 x)-34 f(2 x)+64 f(x)| \leq \frac{25}{2} \phi(0, x)+\phi(x, x)+5 \phi(x,-x)
$$

for all $x \in \mathbb{R}$. Let $g: \mathbb{R} \rightarrow \mathbb{R}$ be a mapping defined by $g(x):=f(2 x)-32 f(x)$ and let

$$
\psi(x)=\frac{25}{2} \phi(0, x)+\phi(x, x)+5 \phi(x,-x)
$$

for all $x \in \mathbb{R}$. In other words, 2.13 means

$$
|g(2 x)-2 g(x)| \leq \psi(x)
$$

for all $x \in \mathbb{R}$. By $(2.5)$, we see that

$$
\sum_{j=0}^{\infty} \frac{1}{2^{j}} \psi\left(2^{j} x\right)<\infty
$$

for all $x \in \mathbb{R}$. Interchanging $x$ into $2^{n} x$ in 2.15 and dividing both sides of 2.15 by $2^{n+1}$, we get

$$
\left|\frac{1}{2^{n+1}} g\left(2^{n+1} x\right)-\frac{1}{2^{n}} g\left(2^{n} x\right)\right| \leq \frac{1}{2^{n+1}} \psi\left(2^{n} x\right)
$$


for all $x \in \mathbb{R}$ and all non-negative integers $n$. We have

$$
\begin{aligned}
\left|\frac{1}{2^{n+1}} g\left(2^{n+1} x\right)-\frac{1}{2^{m}} g\left(2^{m} x\right)\right| & \leq \sum_{j=m}^{n}\left|\frac{1}{2^{j+1}} g\left(2^{j+1} x\right)-\frac{1}{2^{j}} g\left(2^{j} x\right)\right| \\
& \leq \frac{1}{2} \sum_{j=m}^{n} \frac{1}{2^{j}} \psi\left(2^{j} x\right)
\end{aligned}
$$

for all $x \in \mathbb{R}$ and all non-negative integers $n$ and $m$ with $n \geq m$. Therefore we conclude from 2.16 and 2.18 that the sequence $\left\{\frac{1}{2^{n}} g\left(2^{n} x\right)\right\}$ is a Cauchy sequence in $\mathbb{R}$ for all $x \in \mathbb{R}$. Thus, the sequence $\left\{\frac{1}{2^{n}} g\left(2^{n} x\right)\right\}$ is convergent to the mapping $A: \mathbb{R} \rightarrow \mathbb{R}$. Indeed,

$$
A(x):=\lim _{n \rightarrow \infty} \frac{1}{2^{n}} g\left(2^{n} x\right)
$$

for all $x \in \mathbb{R}$. Letting $m=0$ and allowing $n \rightarrow \infty$ in (2.18), we get

$$
|g(x)-A(x)| \leq \frac{1}{2} \sum_{j=0}^{\infty} \frac{1}{2^{j}} \psi\left(2^{j} x\right)
$$

for all $x \in \mathbb{R}$. Using 2.14 in 2.20 , we arrive at the result 2.8). It follows from (2.5), 2.6) and (2.7) that

$$
\left|D_{q} A(x, y)\right|=\lim _{n \rightarrow \infty} \frac{1}{2^{n}}\left|D_{q} A\left(2^{n} x, 2^{n} y\right)\right| \leq \lim _{n \rightarrow \infty} \frac{1}{2^{n}} \phi\left(2^{n} x, 2^{n} y\right)
$$

for all $x, y \in \mathbb{R}$. Therefore the mapping $A$ satisfies 1.3 . By Lemma 2.1, we see that the mapping $A$ is additive. To prove the uniqueness of $\mathcal{A}$, let $T: \mathbb{R} \rightarrow \mathbb{R}$ be another additive mapping satisfying (2.8). We have

$$
\lim _{n \rightarrow \infty} \frac{1}{2^{n}} \sum_{j=0}^{\infty} \frac{1}{2^{j}} \phi\left(2^{n+j} x, 2^{n+j} y\right)=\lim _{n \rightarrow \infty} \sum_{j=n+1}^{\infty} \frac{1}{2^{j}} \phi\left(2^{j} x, 2^{j} y\right)=0
$$

for all $x, y \in \mathbb{R}$ for which $x \in\{x, 0\}$ and $y \in\{x,-x\}$. It follows the above relation and 2.8 that

$$
\begin{aligned}
|A(x)-T(x)| & =\lim _{n \rightarrow \infty} \frac{1}{2^{n}}\left|g\left(2^{n} x\right)-T\left(2^{n} x\right)\right| \\
& \leq \frac{1}{2^{p}} \lim _{n \rightarrow \infty} \frac{1}{2^{n}} \psi\left(2^{n} x\right)=0
\end{aligned}
$$

for all $x \in \mathbb{R}$. So $A=T$. Hence the theorem is proved. 
The upcoming result is a different form of Theorem 2.2 .

TheOREM 2.3. Let $\phi: \mathbb{R} \times \mathbb{R} \rightarrow[0, \infty)$ be a mapping such that

$$
\sum_{j=0}^{\infty} 2^{j} \phi\left(\frac{x}{2^{j+1}}, \frac{y}{2^{j+1}}\right)<\infty
$$

for all $x, y \in \mathbb{R}$ in which $x \in\{x, 0\}$ and $y \in\{x,-x\}$. Suppose that a mapping $f: \mathbb{R} \rightarrow \mathbb{R}$ with $f(0)=0$ satisfies the inequality

$$
\left|\mathcal{D}_{q} f(x, y)\right| \leq \phi(x, y)
$$

for all $x, y \in \mathbb{R}$. Then the limit

$$
A(x)=\lim _{n \rightarrow \infty} 2^{n}\left\{f\left(2 \frac{x}{2^{n}}\right)-32 f\left(\frac{x}{2^{n}}\right)\right\}
$$

exists for all $x \in \mathbb{R}$ and the mapping $A: \mathbb{R} \rightarrow \mathbb{R}$ is a unique additive mapping satisfying

$$
|f(2 x)-32 f(x)-A(x)| \leq \sum_{n=0}^{\infty} 2^{n} \psi\left(\frac{x}{2^{n+1}}\right)
$$

for all $x \in \mathbb{R}$ where $\psi(x)$ is defined in 2.9 .

Proof. Similar to the proof of Theorem 2.2, we have

$$
|g(2 x)-2 g(x)| \leq \psi(x)
$$

for all $x \in \mathbb{R}$, in which $g: \mathbb{R} \rightarrow \mathbb{R}$ is a mapping defined by $g(x):=f(2 x)-$ $32 f(x)$ and $\psi(x)$ is defined in $(2.9)$. It follows 2.22) that

$$
\sum_{j=0}^{\infty} 2^{j} \psi\left(\frac{x}{2^{j+1}}\right)<\infty
$$

for all $x \in \mathbb{R}$. Replacing $x$ by $\frac{x}{2^{n+1}}$ in 2.26 and multiply both sides of 2.26 by $2^{n}$, we get

$$
\left|2^{n+1} g\left(\frac{x}{2^{n+1}}\right)-2^{n} g\left(\frac{x}{2^{n}}\right)\right| \leq 2^{n} \psi\left(\frac{x}{2^{n+1}}\right)
$$


for all $x \in \mathbb{R}$ and all non-negative integers $n$. We have

$$
\begin{aligned}
\left|2^{n+1} g\left(\frac{x}{2^{n+1}}\right)-2^{m} g\left(\frac{x}{2^{m}}\right)\right| & \leq \sum_{j=m}^{n}\left|2^{j+1} g\left(\frac{x}{2^{j+1}}\right)-2^{j} g\left(\frac{x}{2^{j}}\right)\right| \\
& \leq \sum_{j=m}^{n} 2^{j} \psi\left(\frac{x}{2^{j+1}}\right)
\end{aligned}
$$

for all $x \in \mathbb{R}$ and all non-negative integers $n$ and $m$ with $n \geq m$. It follows from 2.27) and 2.29 that for each $x \in \mathbb{R}$ the sequence $\left\{2^{n} g\left(\frac{x}{2^{n}}\right)\right\}$ is a Cauchy. Hence, the mentioned sequence converges for all $x \in \mathbb{R}$. So one can define the mapping $A: \mathbb{R} \rightarrow \mathbb{R}$ by

$$
A(x):=\lim _{n \rightarrow \infty} 2^{n} g\left(\frac{x}{2^{n}}\right)
$$

for all $x \in X$. Putting $m=0$ and tending $n$ to infinity in 2.29 , we obtain

$$
|g(x)-A(x)| \leq \sum_{j=0}^{\infty} 2^{j} \psi\left(\frac{x}{2^{j+1}}\right)
$$

for all $x \in \mathbb{R}$. Therefore, the inequality 2.25 hold. The rest of the proof is same as the proof of Theorem 2.2 .

The following corollaries are the direct consequences of Theorems 2.2 and 2.3 concerning the stability of 1.3 .

COROLlaRY 2.4. Let $\lambda$ be a nonnegative real number with $\lambda \neq 1$ and let $f: \mathbb{R} \rightarrow \mathbb{R}$ be a function satisfies the functional equation

$$
\left|\mathcal{D}_{q} f(x, y)\right| \leq \varepsilon\left(|x|^{\lambda}+|y|^{\lambda}\right)
$$

for some $\varepsilon>0$ and for all $x, y \in \mathbb{R}$. If $g: \mathbb{R} \rightarrow \mathbb{R}$ is a mapping defined by $g(x):=f(2 x)-32 f(x)$, then there exists a unique additive function $A: \mathbb{R} \rightarrow \mathbb{R}$ such that

$$
|g(x)-A(x)| \leq \begin{cases}49 \frac{\varepsilon}{2\left(2-2^{\lambda}\right)}|x|^{\lambda}, & \lambda<1 \\ 49 \frac{2^{\lambda} \varepsilon}{2\left(2^{\lambda}-2\right)}|x|^{\lambda}, & \lambda>1\end{cases}
$$

for all $x \in \mathbb{R}$. 
Proof. In Theorem 2.2 and Theorem 2.3 take $\phi(x, y)=\varepsilon\left(|x|^{\lambda}+|y|^{\lambda}\right)$ for all $x, y \in \mathbb{R}$. By equation (2.8) and (2.25), we obtain the desired result.

CoRollary 2.5. Let $r$ and $s$ be nonnegative real numbers with $\lambda:=r+s \neq$ 1 and let $f: \mathbb{R} \rightarrow \mathbb{R}$ be a function satisfies the functional equation

$$
\left|\mathcal{D}_{q} f(x, y)\right| \leq \varepsilon|x|^{r}|y|^{s}
$$

for some $\varepsilon>0$ and for all $x, y \in \mathbb{R}$. If $g: \mathbb{R} \rightarrow \mathbb{R}$ is a mapping defined by $g(x):=f(2 x)-32 f(x)$, then there exists a unique additive function $A: \mathbb{R} \rightarrow \mathbb{R}$ such that

$$
|g(x)-A(x)| \leq \begin{cases}\frac{6 \varepsilon}{2-2^{\lambda}}|x|^{\lambda}, & \lambda<1, \\ \frac{6 \varepsilon 2^{\lambda}}{2^{\lambda}-2}|x|^{\lambda}, & \lambda>1,\end{cases}
$$

for all $x \in \mathbb{R}$.

Proof. The result follows from the equations $(2.8)$ and $(2.25)$ by defining $\phi(x, y)=\varepsilon|x|^{r}|y|^{s}$.

Corollary 2.6. Let $r$ and $s$ be nonnegative real numbers with $\lambda:=r+s \neq$ 1 and let $f: \mathbb{R} \rightarrow \mathbb{R}$ be a function satisfying the functional equation

$$
\left|\mathcal{D}_{q} f(x, y)\right| \leq \varepsilon\left(|x|^{\lambda}+|y|^{\lambda}+|x|^{r}|y|^{s}\right)
$$

for some $\varepsilon>0$ and for all $x, y \in \mathbb{R}$. If $g: \mathbb{R} \rightarrow \mathbb{R}$ is a mapping defined by $g(x):=f(2 x)-32 f(x)$, then there exists a unique additive function $A: \mathbb{R} \rightarrow \mathbb{R}$ such that

$$
|g(x)-A(x)| \leq \begin{cases}\frac{61 \varepsilon}{2\left(2-2^{\lambda}\right)}|x|^{\lambda}, & \lambda<1 \\ \frac{61 \varepsilon 2^{\lambda}}{2\left(2^{\lambda}-2\right)}|x|^{\lambda}, & \lambda>1\end{cases}
$$

for all $x \in \mathbb{R}$.

Proof. Putting $\phi(x, y)=\varepsilon\left(|x|^{\lambda}+|y|^{\lambda}+|x|^{r}|y|^{s}\right)$ and employing Theorem 2.2 and Theorem 2.3 , we get the result.

We have the following theorem which is analogous to Theorem 2.2. The proof is similar but we bring some parts. 
Theorem 2.7. Let $\phi: \mathbb{R} \times \mathbb{R} \rightarrow[0, \infty)$ be a mapping such that

$$
\sum_{j=0}^{\infty} \frac{1}{32^{j}} \phi\left(2^{j} x, 2^{j} y\right)<\infty
$$

for all $x, y \in \mathbb{R}$ and all $x \in\{x, 0\}$ and $y \in\{x,-x\}$. Suppose that a mapping $f: \mathbb{R} \rightarrow \mathbb{R}$ with $f(0)=0$ satisfies the inequality

$$
\left|\mathcal{D}_{q} f(x, y)\right| \leq \phi(x, y)
$$

for all $x, y \in \mathbb{R}$. Then the limit

$$
\lim _{n \rightarrow \infty} \frac{1}{32^{n}}\left\{f\left(2^{n+1} x\right)-2 f\left(2^{n} x\right)\right\}
$$

exists for all $x \in \mathbb{R}$ and the mapping $Q: \mathbb{R} \rightarrow \mathbb{R}$ is a unique quintic mapping satisfying

$$
\|f(2 x)-2 f(x)-Q(x)\|_{Y} \leq \frac{1}{32} \sum_{n=0}^{\infty} \frac{\psi\left(2^{n} x\right)}{32^{n}}
$$

for all $x \in \mathbb{R}$ where $\psi\left(2^{n} x\right)$ is defined in 2.9 .

Proof. Similar to the proof of Theorem 2.2, one can show that

$$
|h(2 x)-32 h(x)| \leq \psi(x)
$$

for all $x \in \mathbb{R}$, where $h(x)=f(2 x)-2 f(x)$ and $\psi(x)$ is defined in (2.9). The rest of the proof is the same as the proof of Theorem 2.2 .

TheOREM 2.8. Let $\phi: \mathbb{R} \times \mathbb{R} \rightarrow[0, \infty)$ be a mapping such that

$$
\sum_{j=0}^{\infty} 32^{j} \phi\left(\frac{x}{2^{j+1}}, \frac{y}{2^{j+1}}\right)<\infty
$$

for all $x, y \in \mathbb{R}$ and all $x \in\{x, 0\}$ and $y \in\{x,-x\}$. Suppose that a mapping $f: \mathbb{R} \rightarrow \mathbb{R}$ with $f(0)=0$ satisfies the inequality

$$
\left|\mathcal{D}_{q} f(x, y)\right| \leq \phi(x, y)
$$


for all $x, y \in \mathbb{R}$. Then the limit

$$
\lim _{n \rightarrow \infty} 32^{n}\left\{f\left(2 \frac{x}{2^{n}}\right)-2 f\left(\frac{x}{2^{n}}\right)\right\}
$$

exists for all $x \in \mathbb{R}$ and the mapping $Q: \mathbb{R} \rightarrow \mathbb{R}$ is a unique quintic mapping satisfying

$$
|f(2 x)-2 f(x)-Q(x)| \leq \sum_{n=0}^{\infty} 32^{n} \psi\left(\frac{x}{2^{n+1}}\right)
$$

for all $x \in \mathbb{R}$ where $\psi(x)$ is defined in 2.9 .

Proof. The proof is the same as the proof of Theorem 2.3 with $h(x)=$ $f(2 x)-2 f(x)$.

The following corollaries are the direct consequences of Theorems 2.7 and 2.8 concerning the stability of 1.3 . Since the proofs are similar to the previous corollaries, we omit them.

COROLlary 2.9. Let $\lambda$ be a nonnegative real number with $\lambda \neq 5$ and let $f: \mathbb{R} \rightarrow \mathbb{R}$ be a function satisfying the functional equation

$$
\left|\mathcal{D}_{q} f(x, y)\right| \leq \varepsilon\left(|x|^{\lambda}+|y|^{\lambda}\right)
$$

for some $\varepsilon>0$ and for all $x, y \in \mathbb{R}$. If $h: \mathbb{R} \rightarrow \mathbb{R}$ is a mapping defined by $h(x):=f(2 x)-2 f(x)$, then there exists a unique quintic function $Q: \mathbb{R} \rightarrow \mathbb{R}$ such that

$$
|h(x)-Q(x)| \leq \begin{cases}392 \frac{\varepsilon}{32-2^{\lambda}}|x|^{\lambda}, & \lambda<5, \\ 49 \frac{2^{\lambda} \varepsilon}{2\left(2^{\lambda}-32\right)}|x|^{\lambda}, & \lambda>5,\end{cases}
$$

for all $x \in \mathbb{R}$.

Corollary 2.10. Let $r$ and $s$ be nonnegative real numbers with $\lambda:=$ $r+s \neq 5$ and let $f: \mathbb{R} \rightarrow \mathbb{R}$ be a function satisfying the functional equation

$$
\left|\mathcal{D}_{q} f(x, y)\right| \leq \varepsilon\left(|x|^{r}|y|^{s}\right)
$$

for some $\varepsilon>0$ and for all $x, y \in \mathbb{R}$. If $h: \mathbb{R} \rightarrow \mathbb{R}$ is a mapping defined by $h(x):=f(2 x)-32 f(x)$, then there exists a unique additive function $Q: \mathbb{R} \rightarrow \mathbb{R}$ such that 


$$
|h(x)-Q(x)| \leq \begin{cases}\frac{96 \varepsilon}{32-2^{\lambda}}|x|^{\lambda}, & \lambda<5, \\ \frac{6 \varepsilon 2^{\lambda}}{2^{\lambda}-32}|x|^{\lambda}, & \lambda>5,\end{cases}
$$

for all $x \in \mathbb{R}$.

COROLlaRY 2.11. Let $r$ and $s$ be nonnegative real numbers with $\lambda:=$ $r+s \neq 5$ and let $f: \mathbb{R} \rightarrow \mathbb{R}$ be a function satisfying the functional equation

$$
\left|\mathcal{D}_{q} f(x, y)\right| \leq \varepsilon\left(|x|^{\lambda}+|y|^{\lambda}+|x|^{r}|y|^{s}\right)
$$

for some $\varepsilon>0$ and for all $x, y \in \mathbb{R}$. If $h: \mathbb{R} \rightarrow \mathbb{R}$ is a mapping defined by $h(x):=f(2 x)-2 f(x)$, then there exists a unique additive function $Q: \mathbb{R} \rightarrow \mathbb{R}$ such that

$$
|h(x)-Q(x)| \leq \begin{cases}\frac{488 \varepsilon}{32-2^{\lambda}}|x|^{\lambda}, & \lambda<5, \\ \frac{61 \varepsilon 2^{\lambda}}{2\left(2^{\lambda}-32\right)}|x|^{\lambda}, & \lambda>5,\end{cases}
$$

for all $x \in \mathbb{R}$.

The upcoming theorems show that the equation 1.3 is stable under some mild conditions.

ThEOREM 2.12. Let $\phi: \mathbb{R} \times \mathbb{R} \rightarrow[0, \infty)$ be a mapping such that

$$
\sum_{j=0}^{\infty} \frac{1}{2^{j}} \phi\left(2^{j} x, 2^{j} y\right)<\infty
$$

for all $x, y \in \mathbb{R}$ in which $x \in\{x, 0\}$ and $y \in\{x,-x\}$. Suppose that a mapping $f: \mathbb{R} \rightarrow \mathbb{R}$ with $f(0)=0$ satisfies the inequality

$$
\left|\mathcal{D}_{q} f(x, y)\right| \leq \phi(x, y)
$$

for all $x, y \in \mathbb{R}$. Then there exist a unique additive mapping $A: \mathbb{R} \rightarrow \mathbb{R}$ and $a$ unique quintic mapping $Q: \mathbb{R} \rightarrow \mathbb{R}$ such that

$$
|f(x)-A(x)-Q(x)| \leq \frac{1}{30}\left[\frac{1}{2} \sum_{n=0}^{\infty} \frac{\psi\left(2^{n} x\right)}{2^{n}}+\frac{1}{32} \sum_{n=0}^{\infty} \frac{\psi\left(2^{n} x\right)}{32^{n}}\right]
$$

for all $x \in \mathbb{R}$ where $\psi(x)$ is defined in $(2.9)$. 
Proof. By Theorems 2.2 and 2.7, there exist an additive mapping $A_{0}$ : $\mathbb{R} \rightarrow \mathbb{R}$ and a quintic mapping $Q_{0}: \mathbb{R} \rightarrow \mathbb{R}$ such that

$$
\left|f(2 x)-32 f(x)-A_{0}(x)\right| \leq \frac{1}{2} \sum_{n=0}^{\infty} \frac{\psi\left(2^{n} x\right)}{2^{n}}
$$

and

$$
\left|f(2 x)-2 f(x)-Q_{0}(x)\right| \leq \frac{1}{32} \sum_{n=0}^{\infty} \frac{\psi\left(2^{n} x\right)}{32^{n}}
$$

for all $x \in \mathbb{R}$. Therefore it follows from the above inequalities that

$$
\left|f(x)-\left(-\frac{1}{30} A_{0}(x)\right)-\frac{1}{30} Q_{0}(x)\right| \leq \frac{1}{30}\left[\frac{1}{2} \sum_{n=0}^{\infty} \frac{\psi\left(2^{n} x\right)}{2^{n}}+\frac{1}{32} \sum_{n=0}^{\infty} \frac{\psi\left(2^{n} x\right)}{32^{n}}\right]
$$

for all $x \in \mathbb{R}$. So we obtain 2.48 by letting $A(x)=-\frac{1}{30} A_{0}(x)$ and $Q(x)=$ $\frac{1}{30} Q_{0}(x)$ for all $x \in \mathbb{R}$. To prove the uniqueness of $A$ and $Q$, let $A_{1}, C_{1}: \mathbb{R} \rightarrow \mathbb{R}$ be another additive and quintic mappings satisfying (2.48). Put $A^{\prime}=A-A_{1}$ and $Q^{\prime}=Q-Q_{1}$. Hence,

$$
\begin{aligned}
\left|A^{\prime}(x)+Q^{\prime}(x)\right| & \leq|f(x)-A(x)-Q(x)|+\left|f(x)-A_{1}(x)-Q_{1}(x)\right| \\
& \leq \frac{1}{15}\left[\frac{1}{2} \sum_{n=0}^{\infty} \frac{\psi\left(2^{n} x\right)}{2^{n}}+\frac{1}{32} \sum_{n=0}^{\infty} \frac{\psi\left(2^{n} x\right)}{32^{n}}\right]
\end{aligned}
$$

for all $x \in \mathbb{R}$. Since $\lim _{n \rightarrow \infty} \frac{1}{32^{n}} \phi\left(2^{n} x, 2^{n} y\right)=0$, we have

$$
\lim _{n \rightarrow \infty} \frac{1}{32^{n}}\left|A^{\prime}\left(2^{n} x\right)+Q^{\prime}\left(2^{n} x\right)\right|=0
$$

for all $x \in \mathbb{R}$. Thus $Q^{\prime}=0$. Now it follows from 2.48 that $A^{\prime}=0$.

The next theorem is an alternative result of Theorem 2.12.

Theorem 2.13. Let $\phi: \mathbb{R} \times \mathbb{R} \rightarrow[0, \infty)$ be a mapping such that

$$
\sum_{j=0}^{\infty} 2^{j} \phi\left(\frac{x}{2^{j+1}}, \frac{y}{2^{j+1}}\right)<\infty
$$


for all $x, y \in \mathbb{R}$ in which $x \in\{x, 0\}$ and $y \in\{x,-x\}$. Suppose that a mapping $f: \mathbb{R} \rightarrow \mathbb{R}$ with $f(0)=0$ satisfies the inequality

$$
\left|\mathcal{D}_{q} f(x, y)\right| \leq \phi(x, y)
$$

for all $x, y \in \mathbb{R}$. Then there exist a unique additive mapping $A: \mathbb{R} \rightarrow \mathbb{R}$ and a unique quintic mapping $Q: \mathbb{R} \rightarrow \mathbb{R}$ such that

$$
|f(x)-A(x)-Q(x)| \leq \frac{1}{30}\left[\sum_{n=0}^{\infty} 2^{n} \psi\left(\frac{x}{2^{n+1}}\right)+\sum_{n=0}^{\infty} 32^{n} \psi\left(\frac{x}{2^{n+1}}\right)\right]
$$

for all $x \in \mathbb{R}$ where $\psi(x)$ is defined in 2.9 .

Proof. The proof is similar to the proof of Theorem 2.12 and the result follows from Theorems 2.3 and 2.8 .

In the next corollaries, by using Theorems 2.12 and 2.13 , we show that the equation 1.3 can be stable when $\left|\mathcal{D}_{q} f(x, y)\right|$ is controlled by the sum and product of powers of absolute values. Due to similarity of the proofs with the previous corollaries, we present them without proof.

COROLlary 2.14. Let $\lambda$ be a nonnegative real number with $\lambda \neq 1,5$ and let $f: \mathbb{R} \rightarrow \mathbb{R}$ be a function satisfying the functional equation

$$
\left|\mathcal{D}_{q} f(x, y)\right| \leq \varepsilon\left(|x|^{\lambda}+|y|^{\lambda}\right)
$$

for some $\varepsilon>0$ and for all $x, y \in \mathbb{R}$. Then there exist a unique additive mapping $A: \mathbb{R} \rightarrow \mathbb{R}$ and a unique quintic mapping $Q: \mathbb{R} \rightarrow \mathbb{R}$ such that

$$
|f(x)-A(x)-Q(x)| \leq \begin{cases}\frac{1}{30}\left[49 \frac{\varepsilon}{2\left(2-2^{\lambda}\right)}+392 \frac{\varepsilon}{32-2^{\lambda}}\right]|x|^{\lambda}, & \lambda<1, \\ \frac{1}{30}\left[49 \frac{2^{\lambda} \varepsilon}{2\left(2^{\lambda}-2\right)}+392 \frac{\varepsilon}{32-2^{\lambda}}\right]|x|^{\lambda}, & 1<\lambda<5, \\ \frac{1}{30}\left[49 \frac{2^{\lambda} \varepsilon}{2\left(2^{\lambda}-2\right)}+49 \frac{2^{\lambda} \varepsilon}{2\left(2^{\lambda}-32\right)}\right]|x|^{\lambda}, & \lambda>5,\end{cases}
$$

for all $x \in \mathbb{R}$.

COROLlaRY 2.15. Let $r$ and $s$ be positive numbers with $\lambda:=r+s \neq 1,5$ and let $f: \mathbb{R} \rightarrow \mathbb{R}$ be a function satisfying the functional equation

$$
\left|\mathcal{D}_{q} f(x, y)\right| \leq \varepsilon\left(|x|^{r}|y|^{s}\right)
$$


for some $\varepsilon>0$ and for all $x, y \in \mathbb{R}$. Then there exist a unique additive mapping $A: \mathbb{R} \rightarrow \mathbb{R}$ and a unique quintic mapping $Q: \mathbb{R} \rightarrow \mathbb{R}$ such that

$$
|f(x)-A(x)-Q(x)| \leq \begin{cases}\frac{1}{30}\left[\frac{61 \varepsilon}{2\left(2-2^{\lambda}\right)}+\frac{96 \varepsilon}{32-2^{\lambda}}\right]|x|^{\lambda}, & \lambda<1, \\ \frac{1}{30}\left[\frac{6 \varepsilon 2^{\lambda}}{2^{\lambda}-2}+\frac{96 \varepsilon}{32-2^{\lambda}}\right]|x|^{\lambda}, & 1<\lambda<5, \\ \frac{1}{30}\left[\frac{6 \varepsilon 2^{\lambda}}{2^{\lambda}-2}+\frac{6 \varepsilon 2^{\lambda}}{2^{\lambda}-32}\right]|x|^{\lambda}, & \lambda>5,\end{cases}
$$

for all $x \in \mathbb{R}$.

COROLlary 2.16. Let $r$ and $s$ be positive numbers with $\lambda:=r+s \neq 1,5$ and let $f: \mathbb{R} \rightarrow \mathbb{R}$ be a function satisfying the functional equation

$$
\left|\mathcal{D}_{q} f(x, y)\right| \leq \varepsilon\left(|x|^{\lambda}+|y|^{\lambda}+|x|^{r}|y|^{s}\right)
$$

for some $\varepsilon>0$ and for all $x, y \in \mathbb{R}$. Then there exist a unique additive mapping $A: \mathbb{R} \rightarrow \mathbb{R}$ and a unique quintic mapping $Q: \mathbb{R} \rightarrow \mathbb{R}$ such that

$$
|f(x)-A(x)-Q(x)| \leq \begin{cases}\frac{1}{30}\left[\frac{6 \varepsilon}{2-2^{\lambda}}+\frac{488 \varepsilon}{32-2^{\lambda}}\right]|x|^{\lambda}, & \lambda<1, \\ \frac{1}{30}\left[\frac{61 \varepsilon 2^{\lambda}}{2\left(2^{\lambda}-2\right)}+\frac{488 \varepsilon}{32-2^{\lambda}}\right]|x|^{\lambda}, & 1<\lambda<5, \\ \frac{1}{30}\left[\frac{61 \varepsilon \lambda^{\lambda}}{2\left(2^{\lambda^{\lambda}}-2\right)}+\frac{61 \varepsilon 2^{\lambda}}{2\left(2^{\lambda}-32\right)}\right]|x|^{\lambda}, & \lambda>5,\end{cases}
$$

for all $x \in \mathbb{R}$.

Acknowledgements. The authors would like to thank the anonymous referee for a careful reading of the paper and suggesting some related references.

\section{References}

[1] Aoki T., On the stability of the linear transformation in Banach spaces, J. Math. Soc. Japan. 2 (1950), 64-66.

[2] Bodaghi A., Quintic functional equations in non-Archimedean normed spaces, J. Math. Extension 9 (2015), no. 3, 51-63.

[3] Bodaghi A., Moosavi S.M., Rahimi H., The generalized cubic functional equation and the stability of cubic Jordan *-derivations, Ann. Univ. Ferrara 59 (2013), 235-250.

[4] Cădariu L., Radu V., Fixed points and the stability of quadratic functional equations, An. Univ. Timişoara, Ser. Mat. Inform. 41 (2003), 25-48.

[5] Cădariu L., Radu V., On the stability of the Cauchy functional equation: A fixed point approach, Grazer Math. Ber. 346 (2004), 43-52. 
[6] Czerwik S., On the stability of the quadratic mapping in normed spaces, Abh. Math. Sem. Univ. Hamburg. 62 (1992), 59-64.

[7] Hyers D.H., On the stability of the linear functional equation, Proc. Nat. Acad. Sci. USA. 27 (1941), 222-224.

[8] Hyers D.H., Isac G., Rassias Th.M., Stability of functional equations in several variables, Birkhauser, Boston, 1998.

[9] Jung S.-M., Hyers-Ulam-Rassias stability of functional equations in nonlinear analysis, Springer, New York, 2011.

[10] Kannappan P., Functional equations and inequalities with applications, Springer, New York, 2009.

[11] Najati A., Moghimi M.B., Stability of a functional equation deriving from quadratic and additive functions in quasi-Banach spaces, J. Math. Anal. Appl. 337 (2008), 339415.

[12] Park C., Cui J., Eshaghi Gordji M., Orthogonality and quintic functional equations, Acta Math. Sinica, English Series 29 (2013), 1381-1390.

[13] Rassias J.M., On approximation of approximately linear mappings by linear mapping, J. Funct. Anal. 46 (1982), no. 1, 126-130.

[14] Rassias J.M., On approximation of approximately linear mappings by linear mappings, Bull. Sci. Math. (2) 108 (1984), no. 4, 445-446.

[15] Rassias Th.M., On the stability of the linear mapping in Banach spaces, Proc. Amer. Math. Soc. 72 (1978), 297-300.

[16] Rassias Th.M., Brzdęk J., Functional equations in mathematical analysis, Springer, New York, 2012.

[17] Ulam S.M., Problems in modern mathematics, Chapter VI, Science Ed., Wiley, New York, 1940.

[18] Xu T.Z., Rassias J.M., Rassias M.J., Xu W.X., A fixed point approach to the stability of quintic and sextic functional equations in quasi- $\beta$-normed spaces, J. Inequal. Appl. (2010), Article ID 423231, 23 pp, doi:10.1155/2010/423231.

Abasalt Bodaghi

Department of Mathematics

GaRMSAR BRANCH

IslAMic AzAD University

Garmsar, Iran

e-mail: abasalt.bodaghi@gmail.com

Pasupathi Narasimman

Department of Mathematics

Thiruvalluvar University College

of Arts and Science

Gazhalnayaganpatti, Tirupattur-635 901

TAmil Nadu, India

e-mail: drpnarasimman@gmail.com

\author{
Krishnan Ravi \\ Department of Mathematics \\ Sacred Heart College \\ Tirupattur-635 601 \\ TAMILNADU, India \\ e-mail: shckravi@yahoo.co.in
}

\author{
Behrouz Shojaee \\ Department of Mathematics \\ KARAJ BRANCH \\ Islamic Azad University \\ Karaj, Iran \\ e-mail: shoujaei@kiau.ac.ir
}

